\title{
Processos Terapêuticos no Tratamento do Transtorno Afetivo Bipolar: Revisão Integrativa
}

\section{Therapeutic Processes in the Treatment of Bipolar Affective Disorder: Integrative Review Procedimientos Terapéuticos en el Trastorno Afectivo Bipolar: Revisión Integradora}

\author{
Leonardo Oliveira Leão e Silva ${ }^{1}$ \\ Universidade Vale do Rio Doce (Univale) \\ Carlos Alberto Dias \\ Universidade Federal dos Vales do Jequitinhonha e Mucuri (UFVJM) \\ Fernando Ulisses Rosalino \\ Universidade Católica Dom Bosco (UCDB)
}

\begin{abstract}
Resumo
Objetivando identificar na literatura os principais processos terapêuticos no tratamento do Transtorno Afetivo Bipolar (TAB), em suas diversas fases, procedeu-se a uma revisão integrativa de literatura. Utilizando-se as bases de dados Sistema Online de Busca e Análise de Literatura Médica (MEDLINE) e Literatura Latino-Americana em Ciências da Saúde (LILACS), foram selecionados 7 artigos, publicados entre janeiro de 2004 e janeiro de 2014, delimitando-se, a partir destes, dois grandes grupos de abordagem terapêutica: (1) Abordagem farmacológica; (2) Abordagem não-farmacológica. Com base na literatura, a Olanzapina, a ziprasidona e a associação entre haloperidol e prometazina são eficazes no controle da agitação e comportamento violento através da tranquilização após 12 horas. Na prevenção de novos episódios afetivos, nenhuma medicação tem o nível de evidências do lítio. Além disso, no grupo das abordagens não-farmacológicas, os estudos sugerem que a psicoterapia deve ser utilizada em associação com o tratamento farmacológico, pois associados promovem a diminuição na frequência e na duração dos episódios de humor e aumento da adesão à medicação.

Palavras-chave: Transtorno Afetivo Bipolar, terapêutica, adulto
\end{abstract}

\begin{abstract}
In order to identify the literature the main therapeutic methods in the treatment of Bipolar Affective Disorder (BAD), in its various stages, it proceeded to an integrative literature review. Using the MEDLINE and LILACS databases were selected seven articles published between January 2004 and January 2014, is delimiting from these two large groups of therapeutic approach: (1) pharmacological approach; (2) non-pharmacological approach. Based on the literature, olanzapine, ziprasidone, and the association between haloperidol and promethazine are effective in controlling agitation and violent behavior by reassurance after 12 hours. In preventing new affective episodes, no medication has the level of lithium evidence. In addition, the group of non-pharmacological approaches studies suggest that psychotherapy should be used in combination with pharmacological treatment, because they promote the reduction in the frequency and duration of mood episodes and increased medication adherence.

Keywords: Bipolar Affective Disorder, therapeutics, adult

Resumen

Con el fin de identificar en la literatura los métodos terapéuticos principales en el tratamiento de Trastorno Afectivo Bipolar (TAB), en sus diversas etapas, se procedió a una revisión integradora de esa literatura. Así que después de una búsqueda en las bases de datos MEDLINE y LILACS fueron seleccionados siete artículos publicados entre enero de 2004 y enero de 2014, se delimitó a partir de esos dos grandes grupos de enfoque terapéutico: (1) enfoque farmacológico; (2) enfoque no farmacológico. Basado en la literatura, la olanzapina, el ziprasidone, y la asociación entre haloperidol y prometazina son eficaces en el control de la agitación y del comportamiento violento mediante tranquilización después de 12 horas. En la prevención de nuevos episodios afectivos, ningún medicamento tiene el nivel de evidencia del litio. Además, en el grupo de estudios con enfoque no farmacológico, los estudios sugieren que la psicoterapia debe de ser utilizada en combinación con el tratamiento farmacológico, porque promueven la reducción en la frecuencia y en la duración de los episodios del estado de ánimo y un aumento de adherencia a la medicación.

Palabras clave: Trastorno Afectivo Bipolar, terapéutica, adulto
\end{abstract}

${ }^{1}$ Endereço de contato: Rua Miguel Fernandes dos Santos, 398, Vale Verde, Governador Valadares, MG. CEP 35059-600. E-mail: leooliveiraleao@hotmail.com 


\section{Introdução}

Segundo a Organização Mundial de Saúde (2001), 450 milhões de pessoas sofrem de transtornos mentais, resultantes de uma complexa interação de fatores genéticos e ambientais. Os transtornos mentais e do comportamento representam cinco, das dez principais causas de morbidade em todo o mundo. O impacto desses distúrbios foi evidenciado, pois estes representam quatro das dez principais causas de incapacitação. Nesse contexto, o Transtorno Afetivo Bipolar (TAB) afeta cerca de 1,6\% da população e representa uma das principais causas de incapacitação no mundo (Blazer, 2000).

O TAB constitui um transtorno caracterizado por oscilações importantes do humor entre os polos da exaltação ou euforia (mania) e depressão (Sadock \& Sadock, 2007). Os episódios maníacos apresentam grande propensão à recorrência. Cerca de $75 \%$ dos pacientes maníacos têm mais de um episódio, e quase todos os pacientes com episódios maníacos também têm episódios depressivos (Stuart \& Laraia, 2001). Os episódios recorrentes podem causar deterioração do funcionamento, e o número de episódios pode ter um impacto negativo no prognóstico desses indivíduos (Keck Jr., McElroy, \& Arnold, 2001).

O tratamento do TAB envolve manejo nas fases agudas e na terapia de manutenção. Os quadros agudos demandam contenção imediata dos sintomas através da farmacologia e, muitas vezes, internação hospitalar para proteção do paciente. Na fase de manutenção, além da terapia medicamentosa, há indicação do uso de tratamento psicossocial.

Dessa forma, na intenção de identificar os processos terapêuticos a ser utilizados para o $T A B$, delimitou-se como objetivo para o presente estudo identificar na literatura os principais métodos terapêuticos no tratamento do TAB, em suas diversas fases.

\section{Revisão da Literatura}

\section{Considerações Acerca do TAB}

O TAB é um transtorno do humor de longa duração, episódico, potencialmente grave e que, algumas vezes, pode cursar com sintomas psicóticos. A especificidade da doença está associada com elevada morbidade em termos de curso, prejuízo funcional e custo. É uma condição médica contínua para a vida toda, com episódios recorrentes que trazem grande impacto na vida do paciente reduzindo seu funcionamento e sua qualidade de vida (Cipriani, Pretti, Hawton, \& Geddes, 2005).

Considerando o comprometimento medido por meio de vários parâmetros, incluindo funcionamento físico e dias de cama, o transtorno bipolar é responsável por 5\% a 15\% das novas admissões psiquiátricas hospitalares mais prolongadas, consumindo recursos consideráveis dos sistemas de saúde. Estima-se também que o tratamento inadequado seja responsável pela maior parte dos custos do transtorno. Dado o caráter recorrente da doença, constitui a forma mais grave de transtorno do humor. A estimativa de número de episódios ao longo da vida é de 9, em média, e 84\% dos pacientes apresentam mais de 5 episódios, 69\%, mais de 7 e $42 \%$, mais de 11 (Suppes et al., 2009).

O paciente perde mais tempo doente e com menos períodos de remissão relativa que em outras formas de transtorno do humor, por conta da alta taxa de recorrência de episódios na doença bipolar, podendo permanecer doente por períodos consideráveis durante a vida. Os 
que desenvolvem o transtorno por volta dos 20 anos podem perder 9 anos de vida, 12 anos de boa saúde e 14 anos de atividade profissional. Comparado com outros transtornos psiquiátricos, os pacientes bipolares não tratados estão dentre aqueles que apresentam elevado risco de suicídio. O suicídio é tentado por $19 \%$ das pessoas com transtorno do humor bipolar em algum momento de suas vidas (Baldaçara et al., 2011).

A taxa de mortalidade é de duas a três vezes maior que a da população geral, a de divórcios é aproximadamente de duas a três vezes maior comparada à de indivíduos sem o transtorno e a deterioração do estado funcional é o dobro. O estado de mania associa-se a comportamentos inconsequentes e impulsivos que podem produzir ações destrutivas na estabilidade financeira e familiar. Atitudes tomadas sob baixa crítica e julgamento durante o episódio maníaco ou hipomaníaco podem trazer consequências danosas ao indivíduo. As consequências psicossociais do transtorno bipolar afetam não somente o casamento e a ocupação do paciente como também as crianças e outros parentes (Keck, Calabrese Jr., Mcquade, 2006).

Vários estudos mostram uma prevalência ao longo da vida de 0,5\% a 7,5\%. O estudo do Epidemiologic Catchment Area (ECA) apresenta uma estimativa de 1,2\% na faixa etária entre 30 e 40 anos, e o estudo National Comorbidity Survey, de 1,6\%. Para o transtorno bipolar tipo II, a prevalência foi de $0,5 \%$, de acordo com os dados do ECA. A baixa taxa relatada deve-se provavelmente a uma subestimativa (Sadock \& Sadock, 2007).

A prevalência do TAB ao longo da vida é de cerca de $1 \%$ da população geral. É composto por episódios depressivos e maníacos alternados. Afeta principalmente indivíduos mais jovens, e é frequentemente associada com o álcool e a dependência de drogas. Existem dois principais subtipos do TAB: de acordo com o DSM IV-R, o tipo 1 do TAB é caracterizado quando pelo menos um episódio de mania (ou um episódio misto) foi diagnosticado. Já o Tipo 2 do TAB está relacionado a pacientes com duradouros episódios depressivos recorrentes, mas sem nenhum episódio maníaco. Este tipo de TAB afeta as mulheres com mais frequência (Burgess et al., 2001).

O TAB aparece em pacientes que nunca sofreram episódio maníaco, episódio misto ou episódio depressivo grave, mas, em um período de dois anos, demonstraram numerosas crises com alguns sintomas de depressão e sintomas hipomaníacos. A média de idade da pessoa para desenvolver um primeiro episódio (muitas vezes um depressivo) é de 20 anos de idade. Pacientes bipolares não tratados podem desenvolver mais de dez episódios maníacos ou depressivos (Bowden et al., 2010).

Finalmente, em torno de 10 a 20\% dos pacientes, o TAB irá transformar-se em uma forma de ciclo rápido, tanto espontaneamente ou como um resultado de certos tratamentos médicos. Psiquiatras são capazes de iniciar o tratamento com várias estratégias que são mais prováveis de serem eficazes como resultado da identificação de subtipos clínicos da doença bipolar (Vieta et al., 2008).

O estudo longitudinal de Zurique sugere prevalência ao longo da vida de 3,3\%. As variações encontradas podem ser devidas a diferenças nos instrumentos de avaliação diagnóstica utilizados e à dificuldade em obter informações confiáveis. No Brasil, em estudo epidemiológico recente dos transtornos mentais em uma área definida de captação da cidade de São Paulo, a prevalência ao longo da vida para bipolar tipo I foi de 1,0 $\pm 0,3 \%$ (Costa, Cheniaux, Rangé, Versiani, \& Nardi, 2012). 
O transtorno bipolar tipo I geralmente acomete homens e mulheres na mesma proporção, embora no transtorno bipolar tipo II a prevalência seja maior em mulheres. Existem diferenças entre sexos na apresentação do primeiro episódio, em que os sintomas maníacos são mais frequentes em homens, e os depressivos, em mulheres. Não há diferenças de prevalência entre grupos raciais. Seu início é precoce, com picos de aparecimento dos sintomas entre 15 e 19 anos e entre 20 e 24 anos. O primeiro episódio acontece na maioria dos casos (provavelmente em 90\%) antes dos 30 anos de idade. Os primeiros sinais são detectados por volta dos 16 anos e evidências da síndrome completa aparecem por volta dos 19 anos. 0 primeiro tratamento não acontece antes dos 22 anos, e a primeira internação se dá, em média, aos 26 anos. Vários episódios depressivos tendem a ocorrer antes do aparecimento do primeiro episódio de mania. Quando de início tardio (depois dos 60 anos), a doença tende a cursar com caraterísticas menos típicas (história familiar de bipolaridade menos frequente e maior associação com doenças cerebrovasculares) (Blazer, 2000).

Há evidências convincentes de estudos em gêmeos e em famílias de que o transtorno bipolar tem um componente hereditário, embora o modo de transmissão não tenha sido elucidado. Observa-se aumento significativo na frequência de transtorno bipolar do humor em parentes de primeiro grau de pacientes comparados com controles normais (Cipriani, Rendell, \& Geddes, 2010).

O transtorno apresenta alta taxa de comorbidade psiquiátrica, chegando a duas ou mais condições associadas. Dentre elas, o abuso de substâncias é a mais frequente, sendo três vezes mais elevada do que na depressão unipolar, fato que pode confundir o diagnóstico e dificultar a aderência ao tratamento ao longo prazo. Podemos concluir que o transtorno do humor bipolar é uma doença médica crônica que ameaça a vida, é potencialmente progressiva e deteriorante. O tratamento precoce pode alterar seu curso e deve ser mantido a vida toda (Cipriani et al., 2013).

\section{Considerações Acerca do Tratamento Medicamentoso do TAB}

Terapias com o uso de lítio têm sido eficazes e frequentemente usadas para pacientes com mania pura ou exultante e sua profilaxia. No entanto as medicações à base de lítio podem piorar os sintomas depressivos, quando utilizadas para a terapia de manutenção a longo prazo. Além disso, na mania mista, os pacientes do tipo ciclagem rápida e transtorno bipolar associado a abuso de substâncias não respondem bem ao tratamento com lítio (Vieta et al., 2008).

Além da terapia de lítio, ou em lugar de uma terapia de lítio, pode-se reportar o uso frequente de agentes antipsicóticos em relação a pacientes com o TAB durante as fases aguda e de manutenção do tratamento. Agentes antipsicóticos foram utilizados durante quase 40 anos e podem ser usados em combinação com uma terapia com lítio. Antipsicóticos convencionais são eficazes, mas eles podem induzir discinesia tardia, ganho de peso, sedação, depressão e disfunção sexual. Esses efeitos secundários adversos, muitas vezes, levam a não conformidade, em particular, nos casos em que os agentes antipsicóticos são combinados com uma terapia com lítio (Cipriani et al., 2013).

Uma série de abordagens de tratamento somáticas alternativas têm sido relatadas em pacientes que não respondem bem ou que são intolerantes ao tratamento com lítio. Como 
tal, o valproato recebeu a aprovação regulamentar para o tratamento agudo da mania e da carbamazepina foi indicado para esse estado num certo número de países. Uma série de outros anticonvulsivantes (lamotrigina, gabapentina e topiramato) estão sendo testados atualmente (Suppes et al., 2009).

Por causa dos efeitos colaterais dos agentes antipsicóticos convencionais, agentes antipsicóticos atípicos têm sido usados em ensaios e parecem ser de interesse no tratamento do TAB. Atualmente, uma série de estudos prospectivos estão disponíveis com clozapina, risperidona e olanzapina no tratamento do transtorno bipolar. A maioria são estudos de curta duração. Recentemente um estudo randomizado, duplo-cego, controlado por placebo, demonstrou que a clozapina, risperidona e olanzapina podem ser eficazes com efeitos antimaníacos e antidepressivos, tanto como monoterapia e como complemento da terapia de manutenção com lítio ou valproato. A clozapina, risperidona e olanzapina também têm um perfil favorável de efeitos colaterais e um efeito positivo sobre o funcionamento global. Da mesma forma, o valproato, combinado com antipsicóticos, proporciona maior melhoria dos sintomas na mania do que medicamentos antipsicóticos sozinhos e resulta em menor dose do medicamento antipsicótico. Não há atualmente nenhum estudo duplo-cego em relação ao uso de clozapina para transtornos bipolares. No entanto, com base nos resultados de uma série de estudos abertos, a clozapina parece ser eficaz em relação aos pacientes esquizo-afetivos bipolares e incluindo aqueles com ciclos rápidos ou aqueles que respondem inadequadamente a estabilizadores de humor, carbamazepina, valproato ou antipsicóticos convencionais (Macfadden, Alphs, \& Haskins, 2009).

A clozapina parece ser mais apropriada para pacientes bipolares e esquizo-afetivos do que para os esquizofrênicos. Em particular, os estudos mostram que pacientes com estado maníaco e mixe-psicótica da doença possuem melhor resposta medicamentosa em comparação aos demais pacientes com as principais síndromes depressivas. Quatro estudos abertos sugerem a eficácia da clozapina no tratamento de manutenção do transtorno bipolar, e três estudos prospectivos abertos mostram a eficácia da clozapina no estado maníaco da doença. No entanto o número de pacientes nos estudos não foi importante, e estes estudos não são controlados (Keck, Calabrese, \& Mcintyre, 2007).

A clozapina possui alguns efeitos adversos, um dos quais consiste de um grande risco de agranulocitose e, potencialmente, a morte. Além disso, a clozapina demonstrou induzir um ganho de peso significativo, além da sialorreia e efeitos anticolinérgicos significativos. Como resultado, a clozapina não deve ser prescrita em primeiro lugar. Em oposição à clozapina, há relatos de estudos controlados em relação à risperidona e olanzapina. Dois estudos duplo-cegos recentes de mania aguda demonstraram que a olanzapina pode ser mais eficaz do que o placebo. Com base nesses dois estudos, a olanzapina foi recentemente aprovada para a indicação no tratamento da mania (Bowden et al., 2010).

Os efeitos da olanzapina e dival proato no tratamento de mania também foram comparados num grande ensaio clínico aleatório. O grupo de tratamento com olanzapina apresentou uma melhora significativamente maior que a média de classificações de mania e uma proporção significativamente maior de pacientes que alcançaram remissão definida pelo protocolo. Significativamente maior ganho de peso e casos de boca seca, aumento do apetite e sonolência foram relatados com olanzapina, enquanto mais casos de náuseas foram relatados com divalproato. A comparação da olanzapina com lítio para o tratamento da mania também 
tem sido objeto de um estudo duplo-cego randomizado controlado. Esse estudo não mostra diferenças entre as duas drogas. Embora esses estudos apoiem a ideia de que a olanzapina tem efeitos anti-maníacos agudos diretos, vários autores são da opinião de que a olanzapina pode ter propriedades estabilizadoras do humor como profiláticas específicas. A Olanzapina parece ser eficaz no tratamento de manutenção, uma vez que apresentou efeitos em ambos os sintomas: antimaníacos e antidepressivos (Cipriani et al., 2010).

Experimentos têm mostrado que a risperidona pode ser eficaz e segura no tratamento da mania aguda, como um add-on terapia com lítio ou valproato (estudos abertos e dois estudos duplo-cegos controlados) e em monoterapia (estudos abertos). Em uma multicentro, aberta, de estudo de 6 meses, a risperidona foi considerada eficaz e segura como terapia adjuvante, de longo prazo, em bipolar resistente ao tratamento e distúrbios esquizo-afectiva, sem exacerbação de sintomas maníacos. A risperidona teve poucos efeitos colaterais adversos, na sua maioria constituído por ganho de peso. Uma comparação naturalista da clozapina, risperidona e olanzapina no tratamento do transtorno bipolar sugere que a eficácia da tolerabilidade dos três tratamentos é semelhante (Keck et al., 2007).

Um importante fator de diferenciação dessas drogas parece ser o ganho de peso, em particular entre a olanzapina e risperidona. No entanto essa parte pode ser causada pelo uso de agentes estabilizadores do humor. Pacientes com TAB e esquizo-afetivos agora requerem abordagem terapêutica de combinação, devido à natureza cíclica desses distúrbios. Muitos estudos relatam a combinação de agentes estabilizadores do humor com antipsicóticos convencionais e antipsicóticos atípicos. As terapias de combinação produzem um número de efeitos secundários adversos. Os antipsicóticos atípicos (com exceção da clozapina) são agora classificados como agentes de primeira linha para o tratamento da mania adjuvante, porque eles produzem menos efeitos secundários adversos. Os antipsicóticos atípicos são também classificados como agentes de primeira linha para o tratamento combinado de depressão psicótica, e eles são fortemente preferidos quando um antipsicótico é necessário para a manutenção em longo prazo (Keck et al., 2006).

\section{Considerações acerca do tratamento não medicamentoso (Atenção Psicossocial)}

Psicopedagogia com foco no reconhecimento de sinais precoces de recaída é um complemento eficaz para gestão de medicamentos e deve ser oferecido a todos os pacientes com transtorno bipolar. Outras psicoterapias intensivas (terapia cognitivo-comportamental, focada na família, terapia interpessoal e de ritmo social), têm demonstrado benefícios como coadjuvantes na melhora dos sintomas e funções dos pacientes. Para que ela seja efetivada deve ser considerada a disponibilidade financeira daquele que será beneficiado (Miklowitz et al., 2007; Scott, Colom, \& Vieta, 2007).

Os objetivos dos tratamentos psicossociais esperados são: (1) diminuir a negação da doença; (2) aumentar a aderência ao tratamento; (3) diminuir a frequência das crises; (4) meIhorar o relacionamento com familiares; (5) restabelecer o funcionamento ocupacional e social e (6) diminuir o trauma emocional caracterizado por rejeição e estigma. Esses objetivos amplos podem ser atingidos por meio de diferentes metodologias aplicadas isoladamente ou em conjunto, sempre visando à diminuição dos sintomas e à melhora da qualidade de vida do paciente. Assim, a abordagem psicoeducacional surge da necessidade de interlocução 
entre profissionais, pacientes e familiares, para juntos poderem dar sentido à experiência de adoecer, modificando atitudes e trabalhando ativamente na manutenção do bem-estar. Os objetivos dessa abordagem são oferecer informações específicas sobre o transtorno aos pacientes e seus familiares, sobre o que é a doença, o reconhecimento dos sintomas, seus vários tratamentos, a medicação e seus efeitos naturais; aumentar o compromisso com as indicações terapêuticas, diminuindo a frequência, a duração e/ou a intensidade das crises (Miklowitz \& Goldstein, 1997).

Do ponto de vista da família, essa abordagem procura ainda ajudá-la a compreender a pessoa que adoece e trabalhar os sentimentos de culpa, raiva e impotência, determinados pelo membro que adoeceu. Além disso, a experiência de grupo propicia espaço para compartilhar experiências comuns, amenizar o isolamento social tão comum entre essa clientela e diminuir estigmas associados à doença mental. O trabalho psicoeducacional pode ser desenvolvido individualmente ou em grupo de pacientes e/ou familiares, orientado por profissional da área de saúde (médico, psicólogo, enfermeiro dentre outros) que tenha o conhecimento da doença e seja habilitado para o repasse das informações necessárias, para o manejo de dúvidas e acolhimento dos sentimentos que vierem associados ao tema discutido. É comum, nesse tipo de trabalho, o aparecimento de sentimentos ligados à resistência do paciente em aceitar o diagnóstico e o tratamento sugerido. À família cumpre um papel primordial no tratamento desse transtorno. É ela que é capaz de identificar uma crise e deve se mobilizar para procurar assistência e tratamento, até que o paciente tenha condições de cuidar sozinho de si mesmo. Além disso, deve garantir o uso da medicação, manter uma atmosfera em casa de maior tolerância, reduzir as expectativas profissionais do paciente a níveis realistas e encorajá-lo a participar do tratamento e de atividades pouco estressantes (Keila, 1999).

No caso de pacientes com transtorno bipolar, percebeu-se que a medicação psicotrópica melhorava substancialmente o tratamento e o prognóstico dos pacientes, mas não alterava as sequelas psicológicas e sociais da doença, tais como: suicídio, violência, alcoolismo, abuso de drogas, desestruturação familiar, hospitalizações psiquiátricas dentre outras. A eficácia dos novos medicamentos não só permitiu controlar as graves alterações comportamentais das crises de mania e o retraimento e a alienação dos casos de depressão, mas permitiu o tratamento psicológico de pacientes maníaco-depressivos. Esses pacientes puderam, em psicoterapia, centrar sua atenção em problemas psicológicos gerados pela doença, ou desencadeadores desta, assim como enfrentar as tarefas de desenvolvimento por ela interrompidas (Goodwin \& Jamison, 1990). Entende-se por psicoterapia os métodos de tratamento para problemas de natureza emocional, nos quais uma pessoa treinada, mediante a utilização de meios psicológicos, estabelece deliberadamente uma relação profissional com a pessoa que busca ajuda, visando remover ou modificar sintomas existentes, retardar seu aparecimento, corrigir padrões disfuncionais de relações interpessoais, bem como promover o crescimento e o desenvolvimento da personalidade (Wolberg, 1988 ).

Pacientes com transtorno afetivo bipolar costumam, também, apresentar experiências de perdas precoces, insuficiência de gratificações, um ambiente culpabilizante, dificuldade de percepção da realidade, falta de recursos egoicos para lidar com o estresse, e dificuldades nos relacionamentos interpessoais. Esses aspectos podem anteceder o surgimento da doença ou mesmo podem se desenvolver em consequência dela. Outra questão que nem sempre é devidamente enfatizada refere-se às perdas reais e às fantasiadas que ocorrem com o uso 
do lítio ou com outros estabilizadores do humor. Qualquer psicoterapia que pretenda ser efetiva precisa atentar à realidade das percepções positivas que o paciente tem de sua doença (Keila, 1999).

Em relação aos tipos de psicoterapia utilizados no tratamento de pacientes com transtorno bipolar, são poucas ainda as evidências de que haja, por enquanto, maior efetividade e especificidade das diferentes formas de psicoterapia entre si. Dos estudos em andamento, todos com pacientes medicados, estão sendo testadas a psicoterapia interpessoal, a psicoterapia de família de base comportamental (Miklowitz \& Goldstein, 1997) e a terapia cognitiva comportamental. Todas têm apresentado resultados preliminares favoráveis, mas não conclusivos. Psicoterapias psicodinâmicas breves ou de longa duração têm sugerido também resultados interessantes, mas baseados em estudos de casos isolados (Keila, 1999).

\section{Método}

Para o alcance do objetivo, optou-se pela revisão integrativa da literatura, definida como um método por meio do qual resultados de pesquisas são sintetizados, o que possibilita a categorização, análise e avaliação do conhecimento científico já organizado sobre o tema (Ursi, 2005). A síntese realizada por esse método de pesquisa possibilita a contextualização do pesquisador sobre o tema, além de identificar brechas do conhecimento que necessitam ser preenchidas com novos estudos, contribuindo para discussões sobre métodos e resultados de pesquisas anteriores (Polit \& Beck, 2006).

Para a elaboração da revisão integrativa, no primeiro momento o revisor determina o objetivo específico, formula os questionamentos a serem respondidos e, então, realiza a busca para identificar e coletar o máximo de pesquisas primárias relevantes dentro dos critérios de inclusão e exclusão previamente estabelecidos (Beyea \& Nicoll, 1998). Dessa forma, para nortear a realização do presente estudo, formulou-se a seguinte questão: Quais os principais métodos terapêuticos usados no tratamento do TAB?

Para o levantamento bibliográfico deste estudo, realizado no primeiro semestre de 2014, fez-se uma busca on line nas bases de dados Sistema Online de Busca e Análise de Literatura Médica (MEDLINE) e Literatura Latino-Americana em Ciências da Saúde (LILACS). A escolha por essas bases de dados deve-se ao fato de englobarem publicações nacionais e internacionais.

No levantamento dos artigos, tendo em vista sua contextualização com o tema em estudo, foram utilizados como descritores os termos Psicose afetivo-bipolar, Transtorno afetivo-bipolar, Transtorno maníaco-depressivo e Tratamento. Para padronizar a busca de artigos nas bases de dados, foi utilizada como chave a seguinte expressão: (()((Bipolar Disorders[Title/ Abstract]) OR Manic-Depressive Psychosis[Title/Abstract]) OR Bipolar Affective Psychosis[Title/ Abstract]) OR Manic-Depressive Psychoses[Title/Abstract]) AND Treatment[Title/Abstract])].

De acordo com os critérios de inclusão propostos no estudo, os artigos deveriam estar disponíveis nas bases de dados indicadas e estritamente voltados ao objeto de estudo. Deveriam ter sido publicados entre janeiro de 2004 a janeiro de 2014, nos idiomas português, inglês e espanhol, apresentando resumos e textos na íntegra.

Foram identificadas no MEDLINE (A busca foi realizada através da ferramenta de busca PUBMED) 44 referências bibliográficas. Após uma primeira análise baseada nos critérios de 
inclusão, leitura dos resumos disponíveis e avaliação sobre o questionamento elaborado, foram selecionados 6 artigos. No LILACS foram identificadas 59 referências e, seguindo-se os mesmos critérios, foram considerados dois artigos. Ressalta-se que um foi encontrado em ambas as bases de dados, sendo este considerado apenas uma vez no cômputo dos textos incluídos no estudo. Dessa forma, sete artigos fizeram parte da presente revisão.

Após leitura dos artigos selecionados, as informações foram coletadas e digitadas em banco de dados de acordo com as seguintes variáveis: ano, características metodológicas do estudo, autores, objetivos do trabalho, sujeitos estudados - quanto à idade e sexo, resultados encontrados e conclusão/considerações finais do estudo. Todos os registros foram armazenados em um arquivo específico do programa Microsoft ${ }^{\circledR}$ Excel 2007.

\section{Resultados}

Nesta seção, são apresentados os resultados da revisão integrativa, utilizando como pressuposto os artigos selecionados durante o processo de busca. Quanto ao delineamento do estudo, quatro artigos (57\%) eram revisão bibliográfica, dois, (29\%) do tipo Caso-controle, um, (14\%) do tipo qualitativo. Dentre eles, quatro artigos (57\%) estavam estruturados na língua portuguesa, dois, (29\%) na inglesa e um, (14\%) na espanhola. Em relação ao ano de publicação, um artigo (14\%) foi publicado em 2013; um, (14\%) em 2012; dois, (29\%) em 2011; dois, (29\%) em 2005 e um, (14\%) em 2004. A maioria foi publicada no Brasil, seis, (86\%) e os demais na Espanha, um (14\%).

A partir dos textos estudados, foram identificados dois grandes grupos de abordagem terapêutica: Abordagem Farmacológica, três artigos (43\%) e Abordagem não Farmacológica, quatro (57\%). As conclusões dos artigos selecionados foram sumarizados na Tabela 1. Do grupo que trata da abordagem farmacológica para o tratamento do TAB, convém destacar artigos que demonstram o tratamento na fase aguda (mania e hipomania) e a fase de terapia. Já para o grupo da abordagem não farmacológica, há exemplos de artigos que demonstram técnicas e abordagens psicoterápicas para diminuição dos sintomas, sendo sugerida como complemento a abordagem farmacológica, e, em alguns momentos, como uma ferramenta importante na melhora da adesão ao tratamento. 
Tabela 1

Identificação, resultados e conclusões dos estudos selecionados para revisão integrativa. Governador Valadares, 2017.

\begin{tabular}{|c|c|c|c|c|}
\hline AUTOR & $\begin{array}{c}\text { ANO DE } \\
\text { PUBLICACÃO }\end{array}$ & PAÍS & MÉTODO & RESULTADOS/CONCLUSÃO \\
\hline \multicolumn{5}{|r|}{ Abordagem não-Farmacológica } \\
\hline Colom e Vieta & 2004 & Espanha & $\begin{array}{l}\text { Estudo de revisão } \\
\text { da literatura }\end{array}$ & $\begin{array}{l}\text { Afirmam que a psicoeducação deve ser adicionada durante a terapia com estabilizadores de humor, } \\
\text { pois otimiza a eficácia dessa proposta de terapia medicamentosa, além de aumentar a adesão ao } \\
\text { tratamento proposto. }\end{array}$ \\
\hline Costa et al. & 2012 & Brasil & $\begin{array}{l}\text { Estudo caso- } \\
\text { controle (45 } \\
\text { Pacientes) }\end{array}$ & $\begin{array}{l}\text { Os resultados revelaram que a terapia cognitivo-comportamental usada em conjunto com farma- } \\
\text { coterapia para pacientes com transtorno bipolar pode modificar o curso da doença. Ainda obser- } \\
\text { va-se que ocorrem melhorias no humor e qualidade de vida. Afirmam, ainda, que a terapia cogni- } \\
\text { tivo-comportamental de grupo pode ser particularmente útil no tratamento da depressão bipolar. }\end{array}$ \\
\hline Knapp e Isolan & 2005 & Brasil & $\begin{array}{l}\text { Estudo de revisão } \\
\text { da literatura }\end{array}$ & $\begin{array}{l}\text { Afirmam que as abordagens psicoterápicas deveriam ser individualizadas e utilizadas precocemente } \\
\text { no tratamento do transtorno bipolar para melhorar a adesão medicamentosa e ajudar o paciente a } \\
\text { identificar os pródromos da doença com o objetivo de aprender a desenvolver estratégias para lidar } \\
\text { melhor com tais situações, além de terem efeitos nos sintomas residuais os quais estão associados } \\
\text { à cronicidade e a altos níveis de sofrimento e incapacitação. }\end{array}$ \\
\hline Lotufo Neto & 2004 & Brasil & $\begin{array}{l}\text { Estudo de revisão } \\
\text { da literatura }\end{array}$ & $\begin{array}{l}\text { Relatam que o papel da psicoterapia para o tratamento da TAB é enorme e com potencial ainda pouco } \\
\text { explorado. Afirmam que por a TAB ser uma doença crônica, que necessita de acompanhamento e } \\
\text { controle por toda a vida, a psicoterapia seria uma forma de cooperação durante a terapêutica exercida. }\end{array}$ \\
\hline
\end{tabular}

Os resultados revelaram três categorias que descrevem as referidas condições causais: vivendo as crises do transtorno; tendo necessidade do medicamento e convivendo com os efeitos

Miasso et al. 2011 Brasil Estudo qualitativo

colaterais dos medicamentos. Concluem que o desafio frente a terapêutica e sua não adesão relaciona-se à necessidade de mudança de atitude dos profissionais de saúde pois estes mantem o comportamento de culpabilizar o paciente pela interrupção do tratamento

Concluem que a olanzapina, a ziprasidona, o haloperidol/prometazina, o haloperidol/midazolam e

Baldaçara et

al.

2011

Moreno et al.

Estudo duplo-cego o haloperidol isoladamente foram efetivos no controle da agitação e da agressividade secundária (150 Pacientes) e desvantagens, exceto pela associação haloperidol/midazolam que demonstrou os piores resultados em todos os parâmetros. Estudo de revisão Concluem que recentemente novas opções terapêuticas melhoraram o tratamento da mania da literatura mania aguda. 


\section{Discussão}

Observou-se, a partir dos artigos encontrados, que a hipomania e a mania são frequentes. Falhas e erros diagnósticos são comuns, portanto os profissionais da saúde mental, além de clínicos gerais, devem conhecer essas síndromes para evitar demora no diagnóstico e na instituição do tratamento ou sua inadequação. Recentemente novas opções terapêuticas melhoraram o tratamento da mania aguda, principalmente das formas atípicas, porém o lítio continua sendo a primeira opção na mania aguda. Outras incluem o valproato, a carbamazepina e os antipsicóticos atípicos disponíveis, com ênfase na olanzapina, seguida pela risperidona, que possuem o maior número de evidências, levando em conta resultados preliminares da eficácia antimaníaca de aripiprazole, ziprasidona e quetiapina. A oxcarbazepina vem sendo cogitada em substituição à carbamazepina, pressupondo-se eficácia semelhante com melhor perfil de tolerância (Moreno, Moreno, \& Ratzke, 2005 ).

Na prevenção de novos episódios afetivos, nenhuma medicação tem o nível de evidências do lítio. Embora as evidências de eficácia do divalproato e da carbamazepina na prevenção de recorrências permaneçam incertas, ambos são amplamente aceitos como tratamento-padrão para o TAB. Nesse sentido, as pesquisas favorecem a olanzapina como agente alternativo de escolha na terapia preventiva (Moreno et al., 2005 ).

Durante o tratamento da fase aguda da TAB, foi observado, nos artigos selecionados para esta revisão, que a Olanzapina, ziprasidona, haloperidol e prometazina, haloperidol e midazolam e haloperidol são eficazes no controle da agitação e comportamento violento através da tranquilização após 12 horas. Os pacientes que recebem olanzapina, durante essa fase de agitação, tiveram melhores resultados para o controle, uma menor percentagem de sedação excessiva e menor necessidade de restrição mecânica. Já pacientes que recebem a ziprasidona possuem melhores resultados para controle do comportamento agressivo, seguido pelo haloperidol e prometazina. Todas as combinações de fármacos apresentam vantagens e desvantagens, no entanto a combinação de haloperidol e midazolam apresenta os piores resultados quando avaliados os parâmetros de sedação, controle da agitação e violência e efeitos colaterais. Além do custo mais elevado, antipsicóticos atípicos podem ser úteis em casos de emergência (Baldaçara et al., 2011 ).

Durante a fase de terapia medicamentosa, após eventos da fase aguda, alguns autores afirmam existir uma ambivalência em relação à adesão à terapêutica medicamentosa do TAB (Miasso, Cassiani, \& Pedrão, 2011). Segundo eles, o medicamento é colocado em um contexto geral de significações, marcado tanto por aspectos negativos como sofrimento, doença, controle, necessidade, obrigação, hábito e culpa, quanto por aspectos positivos, como possibilidade de convívio com as pessoas, controle das crises, diminuição de reinternações e apoio. Mostra-se, ainda, a evidente tendência à adesão ao medicamento após longa trajetória de crises e internações, quando o paciente já apresenta várias perdas nas diferentes esferas cotidianas. Por outro lado, é fato que muitos dos efeitos colaterais dos medicamentos são transitórios ou podem ser minimizados por diferentes estratégias, sendo fundamental a adequada orientação do paciente e familiar visando à adesão ao tratamento (Miasso et al., 2011).

Embora o tratamento farmacológico seja essencial no tratamento do TAB, ainda há um substancial número de pacientes que, apesar da correta adesão à medicação, permanecem sintomáticos. Alguns dos estudos aqui relacionados, independentemente da abordagem uti- 
lizada, sugerem que a psicoterapia deve ser utilizada em associação com o tratamento farmacológico. As intervenções psicoterápicas apresentam vários benefícios que incluem diminuição na frequência e na duração dos episódios de humor, aumento da adesão à medicação, diminuição nas recaídas e impressões clínicas de melhoras gerais (Knapp \& Isolan, 2005).

As abordagens psicoterápicas devem ser individualizadas e utilizadas precocemente no tratamento do TAB para melhorar a adesão medicamentosa e ajudar o paciente a identificar os fundamentos da doença com o objetivo de aprender a desenvolver estratégias para lidar melhor com tais situações, além de terem efeitos nos sintomas residuais os quais estão associados à cronicidade e a altos níveis de sofrimento e incapacitação (Costa et al., 2012).

Além dessa abordagem, recentemente, o uso da psicoeducação como uma-ferramenta profilática adicional, tem sido reconhecido por várias e prestigiadas diretrizes de tratamento, ampliando e atualizando os paradigmas de tratamento dos transtornos bipolares. Os clínicos devem ter isso presente na prática diária com pacientes portadores do TAB, especialmente porque os benefícios- em termos de menos recaídas e internações- são inquestionáveis e o custo muito baixo. Para isso, há uma necessidade emergencial em treinar profissionais em boas habilidades de comunicação e técnicas psicoeducacionais para implementar em nível mundial sua utilização em transtornos bipolares. A psicoeducação nos dá um potente instrumento não só para melhorar o desfecho de nossos pacientes, mas para auxiliá-los a gerenciar desespero, medos, estigma e baixa autoestima. A psicoeducação deve ser sempre adicionada aos estabilizadores de humor, já que ela otimiza sua eficácia (Colom \& Vieta, 2004).

\section{Considerações Finais}

Com base na literatura, a Olanzapina, ziprasidona, e as combinações de haloperidol/prometazina e haloperidol/midazolam são eficazes no controle da agitação e comportamento violento através da tranquilização após 12 horas; no entanto a combinação midazolam/haloperidol é a que apresenta os piores resultados dentre as demais. Na prevenção de novos episódios afetivos, nenhuma medicação tem o nível de evidências do lítio. Embora as evidências de eficácia do divalproato e da carbamazepina, na prevenção de recorrências, permaneçam incertas, ambos são amplamente aceitos como tratamento-padrão para o TAB. Além disso, os estudos sugerem que a psicoterapia deve ser utilizada em associação com o tratamento farmacológico, pois promovem a diminuição na frequência e na duração dos episódios de humor, aumento da adesão à medicação, diminuição nas recaídas e impressões clínicas de melhoras gerais.

\section{Referências}

Baldaçara , L.; Sanches, M.; Cordeiro, D. C.; \& Jackoswski, A. P. (2011). Rapid tranquilization for agitated patients in emergency psychiatric rooms: A randomized trial of olanzapine, ziprasidone, haloperidol plus promethazine, haloperidol plus midazolam and haloperidol alone. Rev Bras Psiquiatr, 33(1), 30-39.

Beyea, S. C., \& Nicoll, L. H. (1998). Writing an integrative review. AORN Jornal, 67(4), 877-880. Blazer, D. G. Mood disorders: Epidemiology. In B. J. Sadock, \& V. A. Sadock (Ed.), Kaplan \& Sadock's comprehensive textbook of psychiatry (pp. 1298-1308). 7th ed. Philadelphia: Lippincott Williams \& Wilkins. 
Bowden, C. L., Vieta, E., Ice, K. S., Schwartz, J. H., Wang, P. P., Versave, M. (2010). Ziprasidone plus a mood stabilizer in subjects with bipolar i disorder: a 6-month, randomized, placebo-controlled, double-blind trial. J Clin Psychiatry, 71(2), 130-137.

Burgess, S ., Geddes, J., Hawton, K., Townsend, E., Jamison, K., Goodwin, G. (2001). Lithium for maintenance treatment of mood disorders. Cochrane Database Syst Rev, 3,1-41. doi:10.1002/14651858.CD003013

Cipriani, A., Prett y, H., Hawton, K., \& Geddes, J. R. (2005). Lithium in the prevention of suicidal behavior and all-cause mortality in patients with mood disorders: A systematic review of randomized trials. Am J Psychiatry, 162(10), 1805-1819.

Cipriani, A., Rendell, J., \& Geddes, J. R. (2010). Olanzapine in the long-term treatment of bipolar disorder: a systematic review and meta-analysis. J Psychopharmacol, 24(12), 17291738. doi:10.1177/0269881109106900

Cipriani, A., Reid, K., Young, A. H., Macritchie, K., \& Geddes, J. (2013). Valproic acid, valproate and divalproex in the maintenance treatment of bipolar disorder. Cochrane Database Syst Rev., 17(3): CD003196. doi: 10.1002/14651858.CD003196.pub2.

Colom, F., \& Vieta, E. (2004). Melhorando o desfecho do transtorno bipolar usando estratégias não farmacológicas: O papel da psicoeducação. Rev. Bras. Psiquiatr., 26(supl. 3), 47-50.

Costa, R. T., Cheniaux, E., Rangé, B. P., Versiani, M., \& Nardi, A. E. (2012). Group cognitive behavior therapy for bipolar disorder can improve the quality of life. Braz J Med Biol Res, 45(9), 862-868.

Goodwin, F. K., \& Jamison, K. R. (1990). Maniac-depressive illness. New York: University Press. Keck Jr., P. E., Calabrese, J. R., McIntyre, R. S., McQuade, R. D., Carson, W. H., Eudicone, J. M., Carlson, B. X., . . Sanchez, R. (2007). Aripiprazole monotherapy for maintenance therapy in bipolar i disorder: A 100-week, double-blind study versus placebo. J Clin Psychiatry, 68(10), 1480-1491.

Keck Jr., P. E., Calabrese, J. R., McQuade, R. D., Carson, W. H., Carlson, B. X., Rollin, L. M., . . Sanchez, R. (2006). A randomized, double-blind, placebo-controlled 26-week trial of aripiprazole in recently manic patients with bipolar i disorder. J Clin Psychiatry, 67(4), 626-637.

Keck Jr., P. E., McElroy, S. L. \& Arnold, L. M. (2001). Bipolar disorder. Med Clin North Am., 85, 645-661.

Keila, S. B. (1999). Aspectos psicológicos do transtorno afetivo bipolar. Rev. Psiquiatr Clin., 26(3), 123-128.

Knapp, P., \& Isolan, L. (2005). Abordagens psicoterápicas no transtorno bipolar. Rev. Psiquiatr. Clín., 32(supl. 1), 98-104.

Lotufo Neto, F. (2004). Terapia comportamental cognitiva para pessoas com transtorno bipolar. Rev. Bras. Psiquiatr., 26(supl. 3), 44-46.

Macfadden, W., Alphs, L., Haskins, J. T., Turner, N., Turkoz, I., Bossie, C., . . Mahmoud, R. (2009). A randomized, double-blind, placebo-controlled study of maintenance treatment with adjunctive risperidone long-acting therapy in patients with bipolar I disorder who relapse frequently. Bipolar Disord., 11(8), 827-839.

Miasso, A. I.; Cassiani, S. H. B.; Pedrão, L. J. (2011). Transtorno afetivo bipolar e a ambivalência em relação à terapia medicamentosa: analisando as condições causais. Rev. esc. enferm. USP, 45(2), pp. 433-441. 
Miklowitz D. J., Otto M. W., Frank E., Reilly-Harrington, N. A., Wisniewski, S. R., Kogan, . . Sachs, G. S. (2007). Psychosocial treatments for bipolar depression: A 1-year randomized trial from the Systematic Treatment Enhancement Program. Arch Gen Psychiatry. 64(4), 419-426.

Miklowitz, D. J., \& Goldstein, M. J. (1997). Bipolar disorder. A family focused treatment approch. New York: The Guilford Press.

Moreno, R. A., Moreno, D. H., \& Ratzke, R. (2005). Diagnóstico, tratamento e prevenção da mania e da hipomania no transtorno bipolar. Rev. Psiquiatr. Clín., 32(supl. 1), 39-48.

Polit, D. F., \& Beck, C. T. (2006). Using research in evidence-based nursing practice. In Polit D. F., \& Beck C. T. (Eds.), Essentials of nursing research. Methods, appraisal and utilization (pp. 457-494). Philadelphia, USA: Lippincott Williams \& Wilkins.

Sadock, B. J.; \& Sadock, V. A. (2007). Compêndio de psiquiatria: Ciência do comportamento e psiquiatria clínica (9a ed.). Porto Alegre: Artmed.

Scott, J., Colom, F., \& Vieta, E. (2007). A meta-analysis of relapse rates with adjunctive psychological therapies compared to usual psychiatric treatment for bipolar disorders. Int J Neuropsychopharmacol., 10(1), 123-129.

Stuart, G. W., \& Laraia, M. T. (2001). Enfermagem Psiquiátrica: Princípios e prática (D. Batista, Trad.) (6a ed.). Porto Alegre: Artes Médicas.

Suppes, T., Vieta, E., Liu, S., Brecher, M., Paulsson, B., \& Trial 127 Investigators. (2009). Maintenance treatment for patients with bipolar I disorder: results from a north american study of quetiapine in combination with lithium or divalproex (trial 127). Am J Psychiatry, 166(4), 476-488. doi:10.1176/appi.ajp.2008.08020189

Ursi, E. S. (2005). Prevenção de lesões de pele no perioperatório: revisão integrativa da literatura (Dissertação de Mestrado, Universidade de São Paulo, Ribeirão Preto, SP, Brasil).

Vieta, E., Suppes, T., Eggens, I., Persson, I., Paulsson, B., Brecher, M. (2008). Efficacy and safety of quetiapine in combination with lithium or divalproex for maintenance of patients with bipolar I disorder (international trial 126). J Affect Disord, 109(3), 251-263.

Wolberg, L.R. (1988). The technique of psychotherapy (Vol. 1). Filadélfia: Grunet Stratton.

Recebido: 19/05/2015

última revisão: 30/06/2017

Aceite final: 07/07/2017

\section{Sobre os autores:}

Leonardo Oliveira Leão e Silva: Doutor em Saúde Coletiva. Professor da Universidade Vale do Rio Doce. E-mail: leooliveiraleao@hotmail.com

Carlos Alberto Dias: Doutor em Psicologia Clínica. Professor da Universidade Federal dos Vales do Jequitinhonha e Mucuri. E-mail: carlosdiaspsicologo@gmail.com

Fernando Ulisses Rosalino: Mestre em Educação pela Universidade Federal de Mato Grosso do Sul. Psicólogo. Professor da Universidade Católica Dom Bosco. E-mail: rf4680@ucdb.br 